\title{
A Model of Bed Demand to Facilitate the Implementation of Data-driven Recommendations for COVID-19 Capacity Management
}

\section{Teng Zhang}

Stanford University School of Engineering

\section{Kelly McFarlane}

Stanford University Graduate School of Business, Harvard Medical School

Jacqueline Vallon

Stanford University School of Engineering

\section{Linying Yang}

Stanford University School of Engineering

Jin Xie

Stanford University School of Engineering

Jose Blanchet

Stanford University School of Engineering

\section{Peter Glynn}

Stanford University School of Engineering

\section{Kristan Staudenmayer}

Stanford University School of Medicine

Kevin Schulman

Stanford University School of Medicine

David Scheinker ( $\square$ dscheink@stanford.edu )

https://orcid.org/0000-0001-5885-8024

\section{Short report}

Keywords: COVID-19, novel coronavirus disease, model, calculator, bed demand, intensive care, ventilator

Posted Date: June 11th, 2020

DOI: https://doi.org/10.21203/rs.3.rs-31953/v1

License: (c) (1) This work is licensed under a Creative Commons Attribution 4.0 International License.

Read Full License 


\section{Abstract}

Background:We sought to build an accessible interactive model that could facilitate hospital capacity planning in the presence of significant uncertainty about the proportion of the population that is positive forcoronavirus disease 2019 (COVID-19) and the rate at which COVID-19 is spreading in the population. Our goal was to facilitate the implementation of data-driven recommendations for capacity management with a transparent mathematical simulation designed to answer the specific, local questions hospital leadership considered critical.

Methods:The model facilitates hospital planning with estimates of the number of Intensive Care (IC) beds, Acute Care (AC) beds, and ventilators necessary to accommodate patients who require hospitalization for COVID-19 and how these compare to the available resources. Inputs to the model include estimates of the characteristics of the patient population and hospital capacity. We deployed this model as an interactive online tool with modifiable parameters.

Results:The use of the model is illustrated by estimating the demand generated by COVID-19+ arrivals for a hypothetical acute care medical center. The model calculated that the number of patients requiring an IC bed would equal the number of IC beds on Day 23, the number of patients requiring a ventilator would equal the number of ventilators available on Day 27, and the number of patients requiring an $A C$ bed and coverage by the Medicine Service would equal the capacity of the Medicine service on Day 21. The model was used to inform COVID-19 planning and decision-making, including Intensive Care Unit (ICU) staffing and ventilator procurement.

Conclusion:In response to the COVID-19 epidemic, hospitals must understand their current and future capacity to care for patients with severe illness. While there is significant uncertainty around the parameters used to develop this model, the analysis is based on transparent logic and starts from observed data to provide a robust basis of projections for hospital managers. The model demonstrates the need and provides an approach to address critical questions about staffing patterns for IC and AC, and equipment capacity such as ventilators.

\section{Contributions to the literature:}

Generation and implementation of data-driven recommendations for hospital capacity management early in the COVID-19 pandemic

The conceptualization, development, and deployment of an interactive simulation model in two weeks

Data-driven capacity management in the presence of significant uncertainty about the expected volume of patients, their clinical needs, and the availability of the workforce

Trial Registration: Not applicable 


\section{Background:}

As of April 17, 2020 there have been over two million confirmed cases of coronavirus disease 2019 (COVID-19) in over 180 countries, the World Health Organization characterized COVID-19 as a pandemic, and the United States (US) announced a national state of emergency. ${ }^{1-3}$ In parts of China and Italy the demand for intensive care (IC) beds was higher than the number of available beds. ${ }^{4,}{ }^{5}$ The limited availability of testing results in the US make it challenging to estimate the demand for hospital beds that will be generated by COVID-19 patients. We sought to build an accessible interactive model that could facilitate hospital capacity planning in the presence of significant uncertainty about the proportion of the population that is COVID-19 positive (COVID-19+) and the rate at which COVID-19 is spreading in the population. Our approach was to design a tool with parameters that hospital leaders could adjust to reflect their local data and easily modify to conduct sensitivity analyses. Our hypothesis was that datadriven recommendations for capacity management would be more likely to be implemented if they were presented in a transparent mathematical simulation designed to answer the specific questions hospital leadership considered critical. ${ }^{6,7}$

\section{Methods:}

We developed a model to facilitate hospital planning with estimates of the number of Intensive Care (IC) beds, Acute Care (AC) beds, and ventilators necessary to accommodate patients who require hospitalization for COVID-19 and how these compare to the available resources. We deployed this model as an interactive online tool. ${ }^{8}$

Inputs to the model include estimates of the characteristics of the patient population and hospital capacity. The patient population inputs are the starting population of patients without COVID-19 (COVID19-), COVID-19 + patients, and the estimated doubling time for total COVID-19 admissions (i.e. how many days it will take for the number of total admissions at the institution to double). For the patient cohorts, we estimated what percentage of the COVID-19 + patient population would follow 5 potential trajectories through the hospital, assuming various combinations of patient flow between IC and AC. Until more reliable data are available, estimates for input values are generated through review of existing data and discussion with experts. ${ }^{9,10}$ The model will be updated as data become available.

The model produces a daily time series. The first day of the simulation (Day 0) is fixed. For each subsequent day the model uses the projected number of COVID-19 patients, partitions the patients into cohorts, and updates the number of COVID-19 patients requiring IC and AC beds as follows:

1. COVID-19 Admissions: We project the total COVID-19 admissions with an exponential growth model using data based on the first 14 days of patient admissions (The number of total admitted patients up to day $\mathrm{n}$ is the product of the number of patients admitted up to day 0 and 2 to the power of $n$ divided by the doubling time). 
2. Patient Cohorting: The patients are partitioned into 5 care cohorts: Cohorts 1 and 5 are patients who spend time only on, respectively, a General Medicine AC floor and an IC unit. The remaining 3 cohorts are patients who spend time in an IC unit, only before, only after, or both before and after spending time on an AC unit (Table 1).

3. Cohort Length of Stay: Each patient in each cohort spends the number of days specified by the parameter inputs in the IC and AC units. The total census in each of the IC and AC units for each day is calculated as the sum of the patients that arrived in that unit minus the sum of the patients that have been discharged from that unit.

4. Projected IC Bed Requirements: The number of IC beds required each day is the sum of the number of COVID-19 + and COVID-19- IC patients.

5. Projected COVID-19 Medical Team Requirements: The number of patients to be cared for by the Medical Service each day is the sum of the number of COVID-19 + AC patients and COVID-19patients being cared for by the Medicine Service.

6. Projected AC Bed Requirements: The number of $A C$ beds required each day is the sum of the number of COVID-19 + and COVID-19- AC patients.

7. Ventilator Requirements: The number of ventilators required is estimated as the sum of $50 \%$ of nonCOVID-19 IC patients and $100 \%$ of COVID-19 + IC patients.

Table 1

Patient Cohorts and Length of Stay Estimates

\begin{tabular}{|lllllll|}
\hline \multicolumn{2}{|c|}{ COVID Patient Cohorts } & \multicolumn{4}{l|}{ LOS Estimates in Model } \\
\hline Index & Path & $\begin{array}{l}\text { Fraction of Patients } \\
(\%)\end{array}$ & $\begin{array}{l}\text { LOS } \\
\text { Floor }\end{array}$ & $\begin{array}{c}\text { LOS } \\
\text { ICU }\end{array}$ & $\begin{array}{l}\text { LOS } \\
\text { Floor }\end{array}$ & $\begin{array}{c}\text { Total } \\
\text { LOS }\end{array}$ \\
\hline 2 & Floor & 5 & & & 5 \\
\hline 3 & $\begin{array}{l}\text { Floor }->\text { ICU -> } \\
\text { Floor }\end{array}$ & 13.0 & 4 & 9 & 4 & 17 \\
\hline 4 & Floor -> ICU & 1.8 & 6 & 9 & & 15 \\
\hline 5 & ICU - Floor & 13.0 & & 9 & 4 & 13 \\
\hline
\end{tabular}

The model is implemented in R 3.5, RStudio, RShiny 1.4.0 and Python 3.7. The parameters used may be modified as data become available, for use at other institutions, and to generate sensitivity analyses.

\section{Results:}

We illustrate the use of the model by estimating the demand generated by COVID-19+ arrivals for a hypothetical acute care medical center with $100 \mathrm{IC}$ beds, $220 \mathrm{AC}$ beds, 75 ventilators, $80 \%$ occupancy of both IC and AC beds, 1 COVID-19 + IC patient, 2 COVID-19 + AC patients, and a total patient doubling time of 6 days. For COVID-19 + patients, $70 \%$ were assumed to stay only in AC for an average LOS of 5 days 
and $30 \%$ were assumed to spend at least one day in IC with an average IC LOS of 8 days and average AC LOS of 9 days (Table 1 ).

\section{Model projections}

The model calculated that the number of patients requiring an IC bed would equal the number of IC beds on Day 23 (Fig. 1a), the number of patients requiring a ventilator would equal the number of ventilators available on Day 27 (Fig. 1a), and the number of patients requiring an AC bed and coverage by the Medicine Service would equal the capacity of the Medicine service on Day 21 (Fig. 1b).

\section{Sensitivity analyses}

When the doubling time of new admissions is decreased to 3 days (50\%), the number of days until IC and AC hit capacity decrease are, respectively, 11 and 9 . When the doubling time is increased to 12 days (200\%), the number of days until IC and AC hit capacity are, respectively, 51 and 51.

The model was shared publicly for use by other hospitals, and we received feedback from the model from three other institutions.

\section{Discussion:}

In response to the COVID-19 epidemic, hospitals must understand their current and future capacity to care for patients with severe illness. While there is significant uncertainty around the parameters used to develop this model, the analysis is based on transparent logic and starts from observed data to provide a robust basis of projections for hospital managers. The model demonstrates the need to address critical questions about staffing patterns for IC and AC, and equipment capacity such as ventilators, and was used for decision-making in these areas at our institution within two weeks of the project start.

An insight revealed by the model is that under some plausible scenarios, AC may reach capacity before IC and become a bottleneck preventing discharges from IC. In addition to increasing capacity, managers must develop strategies to reduce AC occupancy such as accelerating efforts to discharge patients to convalescent or step-down care such as a hotel or nursing care facility.

The main limitation of this model is the fact that most of the inputs are based on estimates. The epidemiology of COVID-19 is critically important, and ongoing research will update the model. The model is very sensitive to specific aspects of the epidemiology, especially doubling time. The model environment can be easily updated with new parameter data to generate a more precise projection.

\section{Conclusion:}

We describe the conceptualization, development, and deployment of an interactive simulation model to support implementing data-driven hospital capacity management early in the COVID-19 pandemic. The 
model was used to inform decision making within two weeks and in the presence of significant uncertainty about the expected volume of patients, their clinical needs, and the availability of the workforce. Powerful simulation tools to facilitate implementation of data-driven decisions may be applicable across a broad range of settings, including in time-sensitive situations and with relatively little reliable data.

\section{Abbreviations}

COVID-19

coronavirus disease 2019

IC

Intensive Care

AC

Acute Care

ICU

Acute Care Unit

COVID-19+

COVID-19 positive

COVID-19-

COVID-19 negative

\section{Declarations}

\section{Ethics approval and consent to participate:}

Not applicable. This is a mathematical model with no human subjects data.

\section{Consent for publication:}

Not applicable.

\section{Availability of data and materials:}

The model and all underlying inputs supporting the conclusions of this article are available online at: https://surf.stanford.edu/covid-19-tools/covid-19-hospital-projections/

\section{Competing interests:}

The authors declare they have no competing interests.

\section{Funding:}


The authors have no sources of funding to declare for this research.

\section{Authors' Contributions:}

The authors have no sources of funding nor conflicts or other disclosures. TZ, KM, and JV have reviewed all of the data and analyses and take responsibility for their accuracy. TZ, KM, JV, LY, and JX built the model. JB, PG, KrS, KeS, and DS led the conceptualization and design of the model. TZ, KM, KeS, and DS led drafting the correspondence. All authors contributed to critical reading and revision of the correspondence.

\section{Acknowledgments:}

Not applicable

\section{References}

1. Dong E, Du H, Gardner L. An interactive web-based dashboard to track COVID-19 in real time. The Lancet Infectious Diseases Published online February 19, 2020. doi:10.1016/S1473-3099(20)301201.

2. World Health Organization. Coronavirus disease 2019 (COVID-19) Situation Report - 51. World Health Organization. https://www.who.int/docs/default-source/coronaviruse/situationreports/20200311-sitrep-51-covid-19.pdf?sfvrsn=1ba62e57_10. Published March 11, 2020. Accessed March 16, 2020.

3. White House. Proclamation on Declaring a National Emergency Concerning the Novel Coronavirus Disease (COVID-19) Outbreak. White House. https://www.whitehouse.gov/presidentialactions/proclamation-declaring-national-emergency-concerning-novel-coronavirus-disease-covid-19outbreak/. Published March 13, 2020. Accessed March 16, 2020.

4. Wu Z, McGoogan JM. Characteristics of and Important Lessons from the Coronavirus Disease 2019 (COVID-19) Outbreak in China: Summary of a Report of 72314 Cases From the Chinese Center for Disease Control and Prevention. JAMA Published online February 24, 2020. doi:10.1001/jama.2020.2648.

5. Grasselli G, Pesenti A, Cecconi M. Critical Care Utilization for the COVID-19 Outbreak in Lombardy, Italy: Early Experience and Forecast During an Emergency Response. JAMA Published online March 13, 2020. doi:10.1001/jama.2020.4031.

6. Monks T. Operational research as implementation science: definitions, challenges and research priorities. Implementation Sci. 2015;11:81. https://doi.org/10.1186/s13012-016-0444-0.

7. Browman GP, Somerfield MR, Lyman GH, et al. When is good, good enough? Methodological pragmatism for sustainable guideline development. Implementation Sci. 2015;10:28. https://doi.org/10.1186/s13012-015-0222-4. 
8. Medicine SURFS, University S. COVID-19 Hospital ICU and Floor Census Model.

https://surf.stanford.edu/covid-19-tools/covid-19-hospital-projections/ (Accessed March 23, 2020).

9. Zhou F, et al. Clinical course and risk factors for mortality of adult inpatients with COVID-19 in Wuhan, China: a retrospective cohort study. The Lancet. Published online March 11, 2020. doi:https://doi.org/10.1016/S0140-6736(20)30566-3.

10. $10.1056 /$ NEJMoa2002032

Guan Wei-jie, et al. Clinical characteristics of coronavirus disease 2019 in China. NEJM. Published online February 28, 2020. doi:10.1056/NEJMoa2002032.

\section{Figures}

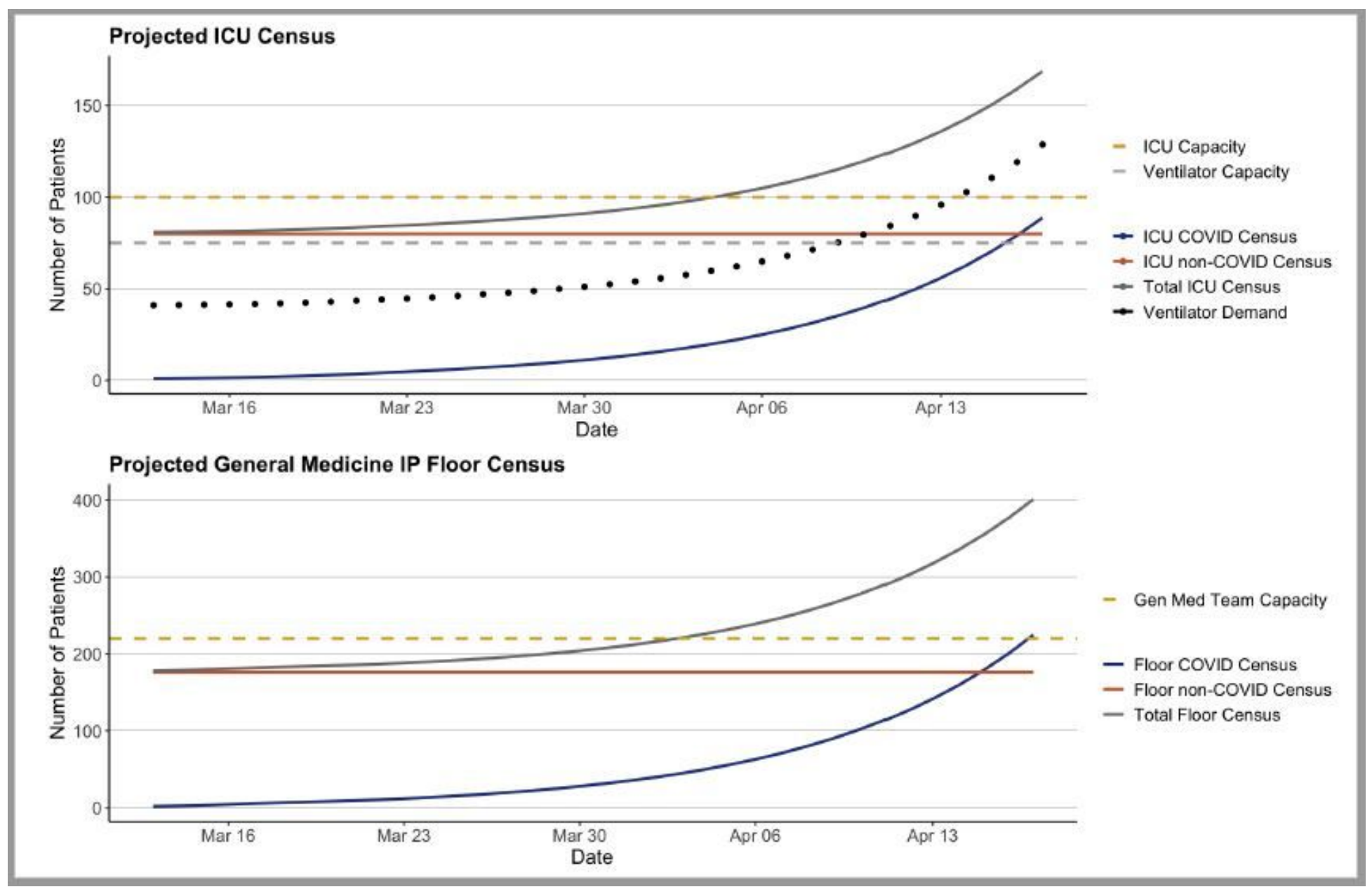

Figure 1

Projected Total ICU and General Medicine Team Acute Care Demand 